\title{
Geoinformation in der kommunalen Abfallentsorgungs - und strategischen räumlichen Planung
}

\author{
Tine Köhler
}

Eingegangen: 6. Mai 2014 / Angenommen: 8. Oktober 2014 / Online publiziert: 28. November 2014

(C) Springer-Verlag Berlin Heidelberg 2014

\begin{abstract}
Zusammenfassung In Gemeinden mit schrumpfender Bevölkerungszahl und in der Folge einer ,Perforierung' der Siedlungsstruktur entwickeln sich die Gebühren für Infrastrukturen, Abgaben und Steuern mehr und mehr zu einer „Zweiten Miete“. Zudem verlieren Immobilien aufgrund der dispersen Schrumpfung der Siedlungen an Wert, wodurch eine Trading-down-Spirale angestoßen werden kann. Um einerseits die Kosten für die Abfallentsorgung zumindest konstant zu halten und andererseits eine kompakte Siedlungsform zu forcieren, sollte trotz Schrumpfung einer dispersen Ausdünnung gegengesteuert werden. In diesem Sinne wird in diesem Beitrag aufgezeigt, wie sich die Kosten für die Sammlung der Restmüllfraktion reduzieren und gleichzeitig die Abfallentsorgung als Steuerungsinstrument für die Siedlungsentwicklung einsetzen ließe. Über den gesamten Prozess hinweg - von der Untersuchung von lokalen Wanderungen, der Verteilung von Wohneinheiten und Innenentwicklungspotenzial innerhalb des Gemeindegebietes bis zur Abfallentsorgungsplanung - wurde auf Geodaten verschiedenster Stellen zurückgegriffen, die zusammen mit unterschiedlichen beschreibenden Daten in einer freien Geoinformationssoftware verarbeitet wurden. Durch Überlagerung der Gesamt- und Fachplanungen lässt sich beispielsweise über eine GIS-gestützte Standort- und Erreichbarkeitspotenzialanalyse von Abfallsammelinseln eine Teilzentralisierung der Abfallentsorgung realisieren. Diese Zentralisierung kann eine ressourcenschonende und strategische Gesamtplanung unterstützen.
\end{abstract}

Dr.-Ing. T. Köhler $(\bowtie)$

ILS - Institut für Landes- und Stadtentwicklungsforschung,

Karmeliterstraße 6,

52064 Aachen, Deutschland

E-Mail: tine.koehler@ils-forschung.de
Schlüsselwörter Geoinformation in der Planung · Abfallentsorgung - Zusammenspiel Fach- und Gesamtplanung · ländlicher Raum · Schrumpfung

\section{Geo-information in waste disposal planning and strategic spatial planning}

\begin{abstract}
The declining growth and the resulting "perforation" of settlements cause high charges for infrastructure use and decreasing municipal tax revenue, which may be regarded as a "second rent". Besides, property values decrease due to vacancy rate, the decay of rural settlements could be initiated. In order to both keep the costs for waste disposal constant as well as to develop a sustainable, compact settlement structure despite of shrinkage, synergies of overall and sectoral spatial planning should be improved. This paper points out a potential strategy to reduce expenses for the cost driver "collection of residual waste" as well as to use the waste disposal as a management tool of the settlement development. Throughout the entire process - from local migration analyses to vacancy rate and possibilities of inner urban development to waste disposal management - geo- and attribute-data were used, provided by different institutions. The data were managed, analysed and visualised by an open source geo-information and geo-database-software. Thus, the information of sectoral and overall spatial planning was overlaid and in result a sustainable, resource-efficient strategic planning is improved using a geo-information tool. Consequently, a centralisation of waste disposal could be improved by a GIS-assisted analysis of location and accessibility of central garbage containers. This centralisation is expected to allow a resource-efficient urban planning.
\end{abstract}


Keywords Geo-information in planning process - Waste disposal management - Synergies of spatial sectoral and overall planning $\cdot$ Rural areas $\cdot$ Declining growth

\section{Einführung}

Während in größeren Städten in schrumpfenden Regionen Stadtumbau und Infrastrukturauslastung bereits umfassend untersucht und Strategien erfolgreich in die Praxis umgesetzt wurden, ist dies für ländliche Regionen noch ein Forschungsrandthema (Beetz 2012: 45 ff.). Entwicklungsinstrumente setzen noch zu stark einseitig auf die Diversifizierung der Landwirtschaft, die Unterstützung der Tourismusfunktion sowie auf den Erhalt des Status-quo anstelle auf Anpassung und Umbau von Strukturen. Zudem folgen in ländlich geprägten Gemeinden Schrumpfung und Siedlungsumbau anderen Logiken, als dies in der Stadt der Fall ist, da andere Eigentumsstrukturen vorherrschen, die ortsbildprägende und identitätsstiftende gebaute Umwelt örtlich begrenzter ist und Funktionsverluste weniger gut kompensierbar und damit, schmerzlicher" sind. Die strategische Planung eines Siedlungsumbaus im ländlich geprägten, peripheren Raum unter Schrumpfungsbedingungen bedarf deshalb einer langfristigen Perspektive, der Unterstützung formeller Planungsinstrumente (für Planungssicherheit und Rechtsverbindlichkeit) sowie einer Nutzung von Synergien verschiedener raumbedeutsamer Planungen.

Die hier beschriebene Forschungsarbeit möchte an einem Beispiel aufzeigen, wie den räumlich-planerischen Herausforderungen durch die Verknüpfung von Siedlungsstruktur und Infrastrukturausstattung begegnet werden könnte. Ziel ist neben dieser allgemeinwohlorientierten Unterstützung der Siedlungskonzentration und der Innenentwicklung der Ortskerne die Verhinderung des Kostenanstiegs beispielsweise für Abfallentsorgung in Räumen mit schrumpfenden Bevölkerungszahlen für den einzelnen Bewohner. Es sollen konkrete Hinweise für ein methodisches Vorgehen von Kommunen bezüglich der Datenverwendung und -zusammenführung zur Unterstützung der langfristigen räumlichen und abfalltechnischen Planung gegeben werden. So sollen an einem Beispiel mithilfe eines Geoinformationssystems durchgeführte Untersuchungen zu Standortpotenzialen und Erreichbarkeitsqualität von Abfallsammelinseln - gemeinsame Container anstelle von einzelnen Abfalltonnen -, Leerstandsrate und -risiko von Häusern und Wohnungen sowie Innenentwicklungspotenzial dargestellt werden, die auch von anderen Kommunen durchgeführt werden können.

In der Forschungsarbeit, die Teil einer Dissertation war (Köhler 2011), wurde außerdem dem Umstand Beachtung geschenkt, dass ein Siedlungsumbau nur unter Einbezug der jeweils Betroffenen funktionieren kann. Durch die räumliche Darstellung von georeferenzierten Meldedaten zusam- men mit Geobasisdaten können den Gebäudeeigentümern und Einwohnern die technischen, wirtschaftlichen und sozialen Folgen von Schrumpfung und mögliche Wirkungen von Maßnahmen sowie deren Zusammenspiel visualisiert und damit verständlicher gemacht werden (Schaffert 2011). So können partizipativ Konzepte erarbeitet werden. Da viele Daten frei verfügbar oder zumindest für die Gemeinden als Träger der Bauleitplanung grundsätzlich nutzbar sind, ist hier eine kostengünstige Möglichkeit der Unterstützung sowohl von Planung als auch von Partizipation vorhanden, wenn Fragen des Datenschutzes und der Zugangsberechtigungen zu sensiblen Daten innerhalb der Verwaltung geklärt sind.

\section{Hintergrund}

Ein erheblicher Bevölkerungsrückgang führt zwangsläufig zu wirtschaftlichen Zwängen und in der Folge zu einer Verschlechterung der infrastrukturellen, sozialen und gesellschaftlichen Strukturen einer Kommune (vgl. Linke/Köhler 2010). Bei einer „flächenhaften Ausdünnung der Besiedlungsstruktur" werden dichte Netze technischer Infrastruktur zunehmend in Frage gestellt (Scherf 2007: 13), da diese schwer ,auf rückläufige Bevölkerungszahlen mit einer an der Kundenzahl orientierten Reduktion der Kosten" reagieren kann (Schiller/Siedentop 2005: 87). So steigen einerseits die Unterhaltungskosten für nicht ausgelastete Infrastruktur, andererseits sinkt die Anzahl derer, die über ihre Gebühren die Infrastruktur finanzieren (demographische Kostenfalle) (vgl. Schmied/Henkel 2007). Dies wurde am Bespiel der Abwasserkanalisationen hinreichend untersucht (u.a. ILS/ Planersocietät 2009). Es lässt sich jedoch auch für den Bereich der Abfallentsorgung, und hier insbesondere für Aufwand und Kosten für Sammlung und Transport, fortführen, da hier die Kosten pro Abfalltonne bei sinkender Anzahl von Abfalltonnen pro $100 \mathrm{~m}$ Straße (Tonnendichte) steigen. Die dazu angestellten Untersuchungen werden in Kap. 3.2 beschrieben.

Neben den Auswirkungen auf die Infrastrukturen ist der Umgang mit dem strukturellen Leerstand ${ }^{1}$ eine Aufgabe der Gemeinden unter Schrumpfungsbedingungen. Struktureller Leerstand ist ein städtebauliches und sozioökonomisches Problem zugleich (vgl. Schmied/Henkel 2007; Kötter 2009), da eine hohe Leerstandsquote einen Attraktivitätsverlust und sinkende Immobilienwerte (Hahne 2013) bedeutet. Zukünftig besonders betroffen sein werden voraussichtlich Wohnbaugebiete der 1960er und 1970er Jahre (Berndgen-

\footnotetext{
${ }^{1}$ Die Ursachen für Leerstände sind vielfältig und komplex (qualitative bauliche Mängel, Mieterwechsel, Arbeiten im Bestand, soziokulturelle Nachfrageveränderungen) und können hier nicht behandelt werden. Es geht hier jedoch um strukturellen, das heißt dauerhaften Leerstand.
} 
Kaiser/Bläser/Fox-Kämper et al. 2014) und kleine Ortsteile ohne Versorgungseinrichtungen. Analysen zu Leerstandsquote und -risiko werden in Kap. 3.3 dargestellt.

Gleichzeitig ist in vielen Gemeinden ein ,Entwicklungsparadoxon' zu beobachten, das heißt die Ausweitung der Siedlungsfläche durch Neubaugebiete auf der einen und die Leerstandsproblematik insbesondere in den Ortskernen auf der anderen Seite. Im Sinne einer nachhaltigen Siedlungsentwicklung ist auf eine Vermeidung perforierter Siedlungen und eine Zentrierung von Wohnnutzung hinzuwirken. In diesem Sinne schließt der vorliegende Beitrag mit der Identifizierung von Innenentwicklungspotenzialen (Kap. 4.1) als Voraussetzung für die Zentrierung sowie der Darstellung von Steuerungsmöglichkeiten durch die Verknüpfung von Abfall- und Siedlungsplanung (Kap. 4.2). Die politische Dimension der Thematik wird in diesem Beitrag zugunsten der Sicht der Geoinformationsverarbeitung und der rein technischen Überlegungen vernachlässigt. Es steht jedoch außer Frage, dass eine Konzepterarbeitung und -umsetzung nur konsensual erfolgen kann (Hagemeister/Haller 2009), was partizipatorische, dialogorientierte, innovationsfördernde Maßnahmen bedingt (Renner 2007: 7). Eine im Sinne von Siebel (2006: 11) nicht-hierarchische Entwicklungsplanung ermöglicht erst die konstruktive Auseinandersetzung der betroffenen Bürger und Eigentümer mit den Erfordernissen, neue Wege in der Schrumpfung zu gehen, und ist Basis für Innovation und Lernprozesse von innen. Top-down diktierte, hoheitliche Maßnahmen werden gerade in einem so sensiblen Bereich wie dem Siedlungsumbau oder auch der Einschränkung der Serviceleistungen im Bereich der Abfallentsorgung kaum im Sinne einer allumfassenden nachhaltigen Siedlungsentwicklung umsetzbar sein.

\section{Untersuchungsschritte und Methodik}

Den Untersuchungsraum bilden vier Ortsteile zweier Gemeinden im südhessischen Odenwaldkreis, die zum Teil sehr peripher gelegen sind, nur über eine Straßenverbindung verfügen (,Sackgassendörfer“) und (fast) reine Wohnstandorte sind. Die Ortsteile wurden, neben dieser aus der Sicht des Abfallmanagements ungünstigen Eigenschaften, aufgrund der hohen Leerstandsraten und -risiken (vgl. Kap. 3.3) ausgewählt. Alle Odenwälder Gemeinden gehören dem Müllabfuhrzweckverband Odenwald (MZVO) an, wodurch sich hinsichtlich der Gebühren einheitliche Rahmenbedingungen ergeben.

In methodischer Hinsicht wurde ein Ansatz gewählt, der auf eine möglichst automatisierte Auswertung zielt. Aufwendige Datenneuerfassungen durch Begehung, wie sie bei Leerstandsuntersuchungen im ländlichen Raum in Deutschland infolge der Abwesenheit eines flächendeckenden Leerstandkatasters noch die Regel sind, wurden des- halb vermieden und nur zur Verifizierung der Ergebnisse stichprobenartig durchgeführt. Stattdessen wurde auf bestehenden Datensätzen aufgebaut, die aus unterschiedlichen Datenquellen zusammengetragen und aufbereitet wurden und die schließlich durch den gemeinsamen Raumbezug die Analyse mittels eines Geoinformationssystems (GIS) erlauben. Dazu wurden frei verfügbare und (gemeindeeigene) vertrauliche Daten integriert, in einer OpenSource (OS)Geo-Datenbank (PostgreSQL/PostGIS) modelliert und in einer ebenfalls OS-GIS-Umgebung (Quantum GIS) verarbeitet und räumlich analysiert.

\subsection{Datengrundlage}

Die räumlichen Auswertungen basieren auf dem Amtlichen Liegenschaftskataster Informationssystem ALKIS, das vom Hessischen Landesamt für Bodenmanagement und Geoinformation (HLBG) für Forschungszwecke zugänglich gemacht wurde.

Für die Auswertung der Abfallentsorgung und ihrer Determinanten (Kap. 3.2) wurden frei verfügbare Sachdaten wie die durchschnittliche Schüttmenge Restmüll pro Kopf sowie die Gebührenhöhe im Untersuchungsgebiet herangezogen. Um die zeitliche Dimension für Sammlung und Transport sowie ein mögliches Einzugsgebiet einer Sammelinsel untersuchen zu können, war zudem eine Unterteilung der Straßenzüge in 100-m-Abschnitte (zur Ermittlung der Anzahl der Abfalltonnen pro $100 \mathrm{~m}$, sogenannte Tonnendichte) und die Darstellung der Erreichbarkeit der Sammelinsel notwendig.

Für die Leerstandsuntersuchungen sowie die Darstellung der Tonnendichte wurde auf Daten der Untersuchungsgemeinden zurückgegriffen. Diese stellten einerseits das aktuelle Melderegister in anonymisierter Form zur Verfügung sowie andererseits die Adressen der Personen mit Anschluss an das Abfallentsorgungssystem (die Zahl der Restmülltonnen war entscheidend). Die als Sachdaten vorliegenden Adressdaten wurden datenbankseitig verknüpft und anschließend geokodiert.

Die Methodik der einzelnen Untersuchungsschritte wird im folgenden Kap. 3.2 konkretisiert und ist für andere Kommunen entsprechend anwendbar.

\subsection{Abfallentsorgung}

Die Abfallentsorgung beinhaltet die Sammlung, den Transport, die Verwertung oder Entsorgung von Abfällen. Dabei können Abfälle im Bring- oder Holsystem gesammelt werden. Bringsysteme können in Form von Sammelinseln oder zentralen Sammelstellen (Recyclinghof) eingerichtet werden, im Holsystem werden die Abfälle direkt dort abgeholt, wo sie anfallen. Die öffentlich-rechtlichen Entsorgungsträger (Kommunen) regeln durch eine Satzung den Anschluss 
der Grundstücke an die Sammelsysteme, Einrichtungen und Anlagen zur Abfallentsorgung und deren Benutzung. Sie regeln ferner, unter welchen Voraussetzungen, in welcher Weise, an welchem Ort und zu welcher Zeit ihnen die Abfälle zu überlassen sind. Dabei kann ein Mindestbehältervolumen oder eine Mindestanzahl von Einsammlungen festgelegt werden.

Der Bereich Sammlung-Umschlag-Transport spielt innerhalb der Abfallwirtschaft eine wichtige, aber häufig unterschätzte Rolle (vgl. Bilitewski/Härdtle/Marek 2000: 675; Förstner 2008). Immerhin verursacht dieser Bereich 30 bis $40 \%$ der Gesamtkosten der Abfallbeseitigung und bietet damit bei Verbesserungen in Organisation und Durchführung erhebliche Einsparmöglichkeiten (Rahmeyer 2004: 21). Transport und Sammlung sind voneinander abzugrenzen, denn der Transport umfasst die Beförderung der Abfallarten zu Deponien, Müllverbrennungsanlagen, Aufbereitungsanlagen, Wertstoffhöfen etc., während die Sammlung alle Vorgänge des Ladens der einzelnen Behälter sowie den Weg zwischen den Ladepunkten beinhaltet (CordLandwehr 2002: 121).

In Räumen mit schrumpfender Bevölkerungszahl, einer großen flächigen Ausdehnung und geringer Bevölkerungsdichte sind trotz konstant bleibender Abfallaufkommen pro Kopf steigende Kosten und damit höhere Gebühren zu beobachten, ${ }^{2}$ da zwar die Zahl der Beitragspflichtigen sowie die Schüttmenge der (gewinnbringenden) Sekundärrohstoffe sinken, die Wegstrecken und die für die Sammlung benötigte Zeit jedoch - beinahe - konstant bleiben. In der Abfallentsorgung ist wie bei den meisten technischen Infrastrukturen die Kostenremanenz sehr groß, da eine sinkende Bevölkerungszahl nicht automatisch sinkende Ausgaben bedeutet. Abfallentsorgung ist somit demographiesensitiv und weist zudem eine sehr geringe Demographieelastizität auf. Bei einer Perforierung der Siedlung steigt beispielsweise die Sammelzeit je Behälter bei weniger als vier Behältern pro $100 \mathrm{~m}$ exponentiell an (Cord-Landwehr 2002: 122), die Entsorgung des Abfalls der verbliebenen Anwohner muss jedoch weiterhin gewährleistet sein.

Der Zusammenhang zwischen Sammlungs- und Transportkosten und der Einwohnerdichte zeigt sich bei der Gegenüberstellung von Daten der Entsorgungszweckverbände, beispielsweise für den Zweckverband Abfallwirtschaft Vogelsbergkreis (ZAV) $145 € / \mathrm{t}$ bei einer Einwohnerdichte von 79 Personen $/ \mathrm{km}^{2}$, den Müllabfuhrzweckverband Odenwaldkreis (MZVO) $103 € /$ t bei 160 Personen $/ \mathrm{km}^{2}$, den Zweckverband Abfallwirtschaft Kreis Bergstraße (ZAKB) $89 € / \mathrm{t}$ bei 374 Personen $/ \mathrm{km}^{2}$ und den Kreis Darmstadt-Dieburg (ZAW) $67 € / t$ bei einer Besiedlungsdichte von 441 Personen $/ \mathrm{km}^{2}$ (vgl. Abb. 1).

\footnotetext{
${ }^{2}$ So auch im Untersuchungsgebiet nach Angaben des Müllabfuhrzweckverbandes Odenwald (MZVO).
}

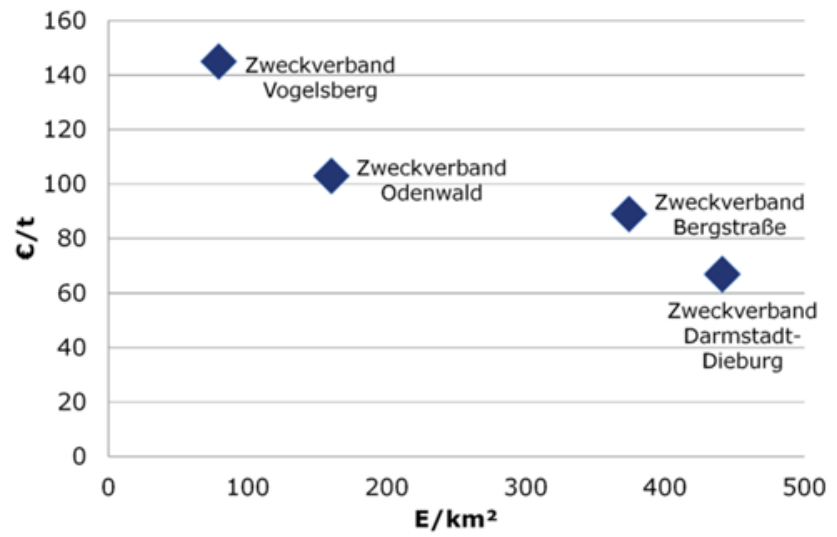

Abb. 1 Relation zwischen Sammlungs- und Transportkosten und Einwohnerdichte. (Quelle: Eigene Berechnung auf der Grundlage der Einwohnerzahlen des Hessischen Statistischen Landesamtes (2013: 12), der Gebührenordnungen und Satzungen der Abfallzweckverbände sowie Hessischer Rechnungshof (2009: 47))

Als weiterer kostenbestimmender Faktor ist die Abfallmischung zu nennen, weil die Abfallfraktionen (Restmüll, Grünschnitt, Altholz, Metalle etc.) unterschiedliche Entsorgungswege haben und die Verwertbarkeit stark differiert. Vergleicht man die Kosten pro Tonne Abfall und deren fraktionelle Anteile von Rest-, Biomüll und Altpapier, ergibt sich der Restmüllanteil trotz Reduzierung des Aufkommens durch Eigenkompostierung als kostenbestimmender Faktor. ${ }^{3}$ So ist beispielsweise von Art und Menge der Restmüllfraktion neben der Auslastung auch der energetische Output der Müllverwertungsanlage abhängig (Miofsky/Souren 2010: 7). Bei der Erarbeitung möglicher Kosteneinsparkonzepte sollte ein besonderes Augenmerk demnach auf der Restmüllfraktion liegen.

Neben der Reduzierung des Restmülls ist in den letzten zehn Jahren in ganz Deutschland das Abfallaufkommen pro Kopf insgesamt deutlich gesunken. ${ }^{4}$ Maßnahmen zur Änderung im Abfallverhalten sind wegen des (im ländlichen Raum besonders hohen) Erfassungsgrades ${ }^{5}$ (Salhofer 2001: 126) nicht erforderlich. Vielmehr sind Versuche zu unternehmen, die Fixkosten in der Entsorgung des Restmülls zu senken. Bilitewski (2007: 48) gibt allein für die Sammlung des Restmülls einen 23-prozentigen Anteil an den Abfallgebühren in Deutschland an.

Die aufgezeigte Abhängigkeit der Pro-Kopf-Kosten und der Einwohnerdichte zeigt, dass sich eine Kostenreduktion durch eine räumliche Konzentration des Einsammelns errei-

\footnotetext{
${ }^{3}$ Daten des Hessischen Rechnungshofes (2009), eigener Vergleich der Daten der Abfallzweckverbände Vogelsbergkreis, Darmstadt-Dieburg, Bergstraße und Odenwaldkreis.

${ }^{4}$ Zwischen 2002 und 2005 um 18\% (Statistisches Bundesamt 2014).

${ }^{5}$ Der Erfassungsgrad gibt den Anteil an, mit dem ein getrennt zu sammelnder Altstoff tatsächlich erfasst wird, ohne mit anderen Stoffen vermischt zu sein, z. B. getrennte Altpapiersammlung.
} 
chen ließe. Zudem benötigt ein Entsorgungsunternehmen gewisse Volumina an Abfall, um wirtschaftlich arbeiten zu können. Um dies zu erreichen, kann zwischen Bringund Holsystem unterschieden werden. Als Kriterium für das Holsystem wird im Allgemeinen eine Mindestanzahl an Entleerungen bei einer gegebenen (kleinstmöglichen) Behältergröße herangezogen (Salhofer 2001: 130). Für Altstoffe wurde bei einer Behältergröße von 601 ein Entleerungsintervall von 13 Entleerungen pro Jahr und für biogene Reststoffe mindestens 39 Entleerungen pro Jahr errechnet, um sowohl für Nutzer als auch für Sammler ein praktikables Verhältnis zu erreichen. Ergibt sich rechnerisch aufgrund nicht ausreichender anfallender Mengen eine geringere Anzahl an Entleerungen, ist die betreffende Abfallart für die Sammlung im Holsystem aus der Sicht des Abfallmanagements nicht geeignet und eine Umstellung auf das Bringsystem zu diskutieren. Diese Diskussion ist gegebenenfalls gemeinsam mit den Betroffenen zu führen, da eine rein rechnerische Betrachtung die tatsächlichen Gegebenheiten und Bedürfnisse nicht abbilden kann und allein noch keine Systemveränderung begründet. Zudem werden die Einwohner über die Beteiligung möglichst frühzeitig sensibilisiert, womit ein wichtiger Grundstein für die Akzeptanz der Maßnahme gelegt wird (vgl. IÖR 2001).

Fußläufig erreichbare Sammelinseln, die gegenüber den zentralen Sammelstellen den größeren Komfort für Nutzer bieten, eignen sich jedoch nicht für jedes Abfallaufkommen. Für die Berechnung der maximalen Entfernung für Sammelinseln in Abhängigkeit zur Siedlungsdichte muss eine Mindestgröße der Behälter und ein praktikables Entleerungsintervall zugrunde gelegt werden. Aus der Gegenüberstellung der pro Einwohner durchschnittlich anfallenden und der entleerten Volumina ergibt sich eine Anzahl an Einwohnern im Einzugsbereich der Sammelinsel. Unter Annahme einer gleichmäßigen Verteilung der Einwohner im Gebiet wird diese Einwohnerzahl auf die Siedlungsdichte in einer Fläche (Gemeinde oder Landkreis) umgelegt. Der Radius des entstehenden Kreises stellt die maximale Entfernung zu einer Sammelinsel dar (vgl. Abb. 2).

Die Unschärfe, welche die Annahme einer gleichmäßigen Verteilung von Einwohnern mit sich bringt, kann von Gemeinden mithilfe eines GIS vermieden werden. Zur Verdeutlichung wurde beispielhaft für einen Ortsteil, in dem die Abfallsammlung aufgrund geringer Behälterdichte und enger Gassen ohne Wendemöglichkeit mit hohem logistischem Aufwand verbunden ist, eine Sammelinsel vorgeschlagen und mithilfe einer Erreichbarkeitsanalyse der Einzugsbereich der Sammelinsel festgestellt (vgl. Abb. 3). Letzteres erfolgte über eine Kürzeste-Wege-Routinganalyse. Einbezogen wurden dabei außerdem ein digitales Geländemodell, um topographische Gegebenheiten berücksichtigen zu können, da steile Anstiege insbesondere für weniger mobile Personen schlechter zu bewältigen sind als

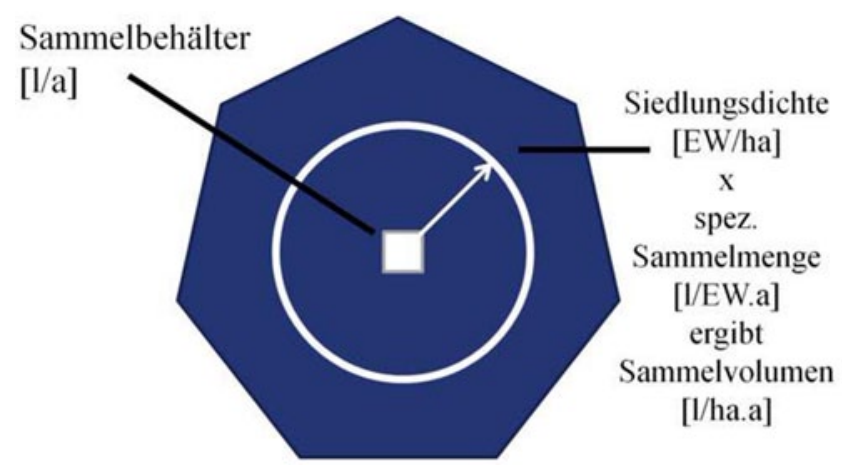

Abb. 2 Einzugsgebiet von Sammelinseln (Systemskizze). (Quelle: Eigener Entwurf in Anlehnung an Salhofer (2001: 134))

ebene Strecken. Als Entfernung wurde zunächst in Anlehnung an die empfohlene Erreichbarkeit von Haltestellen des öffentlichen Personennahverkehrs von $600 \mathrm{~m}$ bzw. maximal zehn Minuten Gehzeit (Prinz 1999: 71; OREG 2012: 31) angenommen, wobei aufgrund des mitzuführenden Restmülls keine Überschreitung der 600-m-Distanz zugelassen wurde. So ergibt sich die für einen wirtschaftlichen Betrieb einer Sammelinsel erforderliche Siedlungsdichte. Zur zusätzlichen Berücksichtigung der Steigungen auf der Grundlage des digitalen Höhenmodells wurde die Routinganalyse mithilfe eines Knoten-Kanten-Modells, ausgehend von dem Knoten, auf dem die Sammelinsel geplant ist, mit einer Begrenzung der Gesamtkosten (Schwellenwert 500) durchgeführt. Für die Berechnung der Gesamtkosten wurde neben dem Abstandsgewicht auch die Steigung (je Kante) durch Einführung von Steigungsgewichten berücksichtigt.

\subsection{Verteilung der Wohneinheiten im Raum}

Die Untersuchung stellt eine Status-quo-Analyse dar. Für eine nachhaltige Planung müssen jedoch auch zukünftige Entwicklungen einbezogen werden. Dazu muss eine Annahme der zukünftigen Verteilung von Wohneinheiten im Gemeindegebiet getroffen werden. Da dies auf der Gemeindeebene mit großen Unsicherheiten verbunden ist, wurde dabei einerseits eine Mixed-Method-Untersuchung für eine Gemeinde vorgenommen, bei der die zunächst durchgeführte quantitative Datenauswertung durch Ortsbegehungen und Expertengespräche verifiziert wurde. Andererseits wurden zum Teil aggregierte Aussagen für Wohngebiete (anstelle von einzelnen Gebäuden) getroffen.

Für die Verteilung von genutzten Wohneinheiten im Raum sind Sterbefälle, Wegzüge und innerörtliche Umzüge von Interesse. Diese können die Gemeinden anhand der amtlichen Melderegister einsehen. Aus datenschutzrechtlichen Gründen ist dies Dritten nicht möglich. Im Forschungsprojekt lagen jedoch amtliche Melderegister über einen Zeitraum von zehn Jahren in anonymisierter Form vor, einschließlich der gebäudebezogenen Daten über Zuzug, Weg- 
Abb. 3 Sammelinsel und Angabe der fußläufigen Erreichbarkeit. (Quelle: Eigene Darstellung auf Basis der ALKIS-Daten des Hessischen Landesamtes für Bodenmanagement und Geoinformation (HLBG))

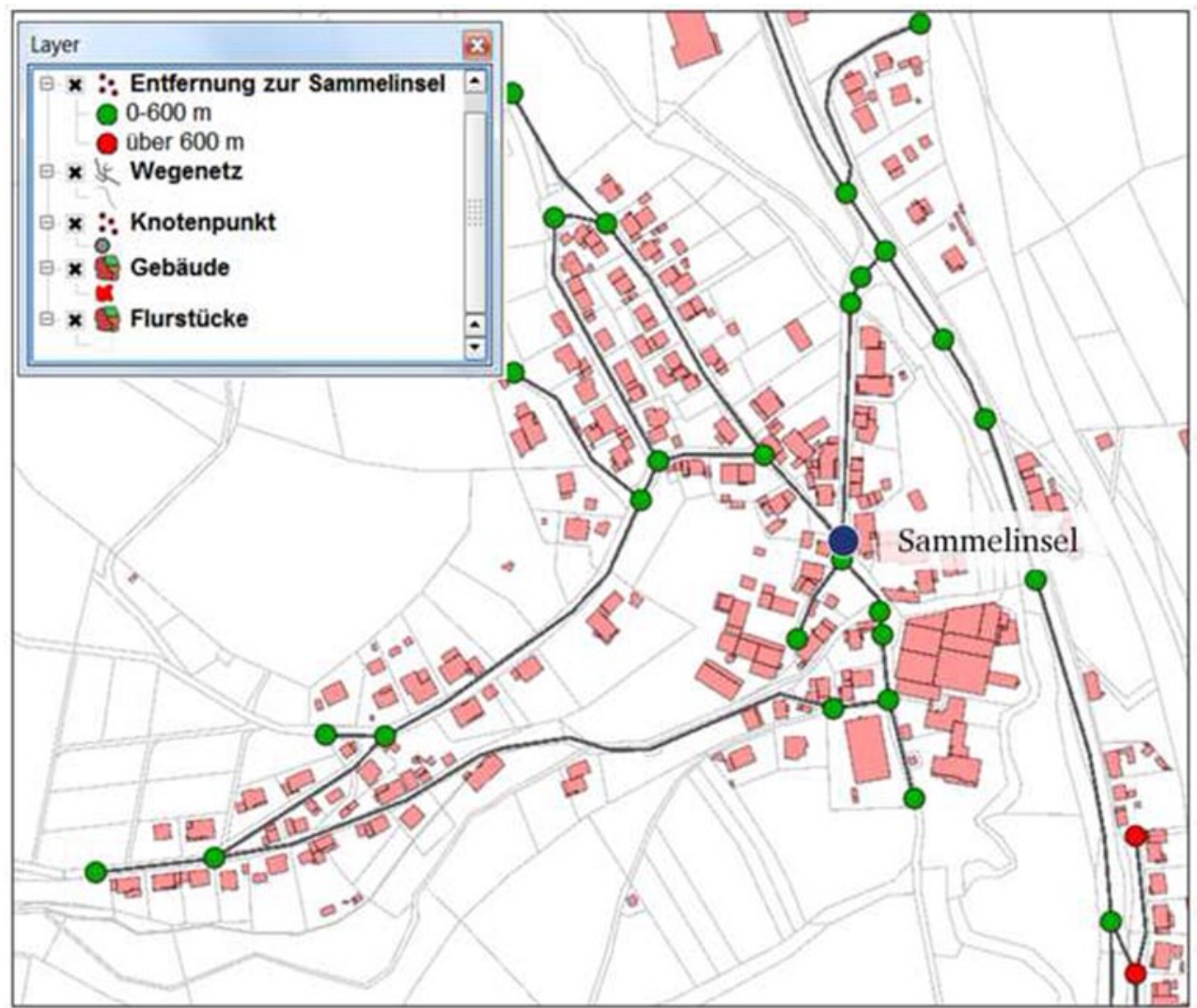

zug oder Tod eines Bewohners. Die Daten wurden über einen Geokodierungsdienst des Hessischen Landesamtes für Bodenmanagement und Geoinformation (HLBG) georeferenziert, welcher die Hauskoordinaten im amtlichen Koordinatensystem aus dem ALKIS zurückgibt.

Für eine Aussage über die zukünftig zu erwartende Verteilung im Raum wurde das gesamte Siedlungsgebiet der Untersuchungsgemeinden in Baualtersklassen unterteilt, um die Wanderungsbewegungen zwischen den Baugebieten untersuchen zu können (vgl. Kap. 4). Dabei konnte neben den Auswertungen des Schwarzplans auf Unterstützung eines ortsansässigen Experten (Vertreter des Regionalmuseumsvereins) zurückgegriffen werden. Der Experte bekam über den Web-Map-Service (WMS) des Hessischen Landesamtes für Bodenmanagement und Geoinformation die Automatisierte Liegenschaftskarte (ALK) als Kartengrundlage zur Verfügung gestellt, in der er mithilfe der OS-Software Quantum GIS nach kurzer Einweisung selbst Baualtersklassen als Flächen einzeichnen konnte. Die Daten des Mikrozensus 2010 eignen sich für eine solche Untersuchung nicht, da sie zwar für weite Teile des Bundesgebietes eine Einteilung in Baualtersklassen enthalten, nicht jedoch für Gemeinden des ländlichen Peripherraumes.

Als weitere Informationsquelle konnten die Bauleitpläne der Untersuchungsgemeinden herangezogen werden, die über die kommunale Geodateninfrastruktur (GDI) abrufbar sind.
Neben der Verteilung der Wohneinheiten wurde die damit direkt in Zusammenhang stehende Leerstandsrate eruiert und eine Einschätzung zur Entwicklung der Leerstandsquote vorgenommen. Die Leerstandsquote lässt sich auf der Grundlage des datenbankseitig mit den Geobasisdaten verknüpften Melderegisters feststellen und mithilfe eines GIS visualisieren: Wird das Melderegister der Kommunen zugrunde gelegt, lassen sich mithilfe der Altersangaben und der Anzahl der Bewohner pro Haus Wohnbaugebiete mit hoher Leerstandsrate ermitteln. Zur Prognose, welche Gebäude innerhalb eines bestimmten Zeitraumes leerfallen (Leerstandsrisiko), werden Gebäude mit nur einem verbliebenen Bewohner im Hochbetagtenalter abgefragt. Im Vergleich mit dem statistisch anzunehmenden Todesjahr der Person kann der Zeithorizont bis zum Leerfallen einer Immobilie abgeschätzt werden. Ob diese Immobilie jedoch auch von strukturellem Leerstand betroffen sein wird, beeinflussen unterschiedlichste Kriterien der Nachfrage. Dazu gehören direkte Einflussfaktoren wie Preisgestaltung, Bauzustand, Wohnumfeld, Eigentumsverhältnisse, Marktverhalten der Eigentümer, aber auch indirekte wie mögliche finanzielle Förderungen eines Umbaus oder einer Renovierung, Arbeitsmarkt, gesellschaftliche Rahmenbedingungen, die soziale Struktur des Umfelds und Identifikation damit (vgl. Köhler 2011; Schmied 2007: 12 ff.; Schöfl/Schöfl/ Speidel 2009: 273 ff.). 


\section{Ergebnisse}

Die empirischen Untersuchungen zu innerörtlichen Wanderungen und der Leerstandsproblematik in vier peripher gelegenen Ortsteilen zweier Gemeinden mit etwa 13.000 bzw. 9000 Einwohnern im Odenwaldkreis zeigen, dass insgesamt ein Trend zur Konzentration der Bevölkerung in der jeweiligen heutigen Kerngemeinde vorliegt. Diese (Re)Zentrierung ist aus infrastrukturtechnischer und hygienischer (Korrosions- und Verkeimungsrisiko bei langen Standzeiten von Abwasser bzw. Trinkwasser) - und damit letztlich finanzieller - Sicht zu begrüßen, da so kompakte Siedlungen entstehen (Linke/Köhler 2010).

Da sich bei der automatisierten Leerstandsuntersuchung Unsicherheiten ergeben (bezüglich Leerstandsdauer, Korrektheit der amtlichen Geobasisdaten, tatsächliche Nutzung des Gebäudes, Fehler im Melderegister etc., vgl. Köhler 2011), wurden die Erkenntnisse mit qualitativen Untersuchungen ergänzt. Dazu gehörten Ortsbegehungen, Gespräche mit Ortskundigen und Experten aus Verwaltung und Politik. So konnte beispielsweise eine in den ALKIS-Daten als Wohnnutzung dargestellte Siedlung, die nach Verschneidung mit den Melderegisterdaten komplett leer stehen müsste, als Ferienhausgebiet identifiziert werden. Eine Verifizierung ist daher unerlässlich.

In Bezug auf die Standort- und Erreichbarkeitsuntersuchungen von Sammelinseln sind zwei unterschiedliche Ergebnisse möglich: Entweder kann eine Entsorgung über eine Sammelinsel wegen der Erreichung der notwendigen Schüttmenge durch eine ausreichende Einwohnerdichte im Einzugsbereich der Insel empfohlen werden oder aber die Einwohnerdichte reicht nicht aus und eine Sammelinsel kann nicht sinnvoll platziert werden (vgl. Kap. 4.2). Im in Abb. 3 dargestellten Beispiel ist die Sammelinsel von 99\% der Einwohner über einen Fußweg von unter den als Obergrenze festgelegten $600 \mathrm{~m} \mathrm{bzw}$. zehn Minuten (vgl. Kap. 3.2) erreichbar, wobei - bei konstantem Pro-KopfAufkommen - die notwendige Schüttmenge erreicht würde. Mit dieser Lösung wäre gleichzeitig der Spagat zwischen möglichst wendigen (kleinen) Fahrzeugen für die Sammlung in schmalen Straßen und Sackgassen und möglichst großen Fahrzeugen zur Einsparung mehrfacher An- und Abtransporte (Fahrten zwischen Betriebshof und Sammelgebiet) (Cord-Landwehr 2002: 125) gelungen, da die Sammelinsel an einer Stelle aufgestellt werden würde, die ein Rangieren mit großen Fahrzeugen zuließe.

Für die Untersuchung des Zusammenhangs zwischen Umzügen und Leerstand wurden Bewohner eines Baugebietes der 1990er Jahre am Rande der Kerngemeinde (98\% Einheimische) zu deren vorherigen Wohngebäuden zugeordnet, wobei eine innerörtliche Wanderung insbesondere aus der Baualtersklasse vor 1919 in das neuere Baugebiet zu beobachten war (vgl. Abb. 4 und 5).

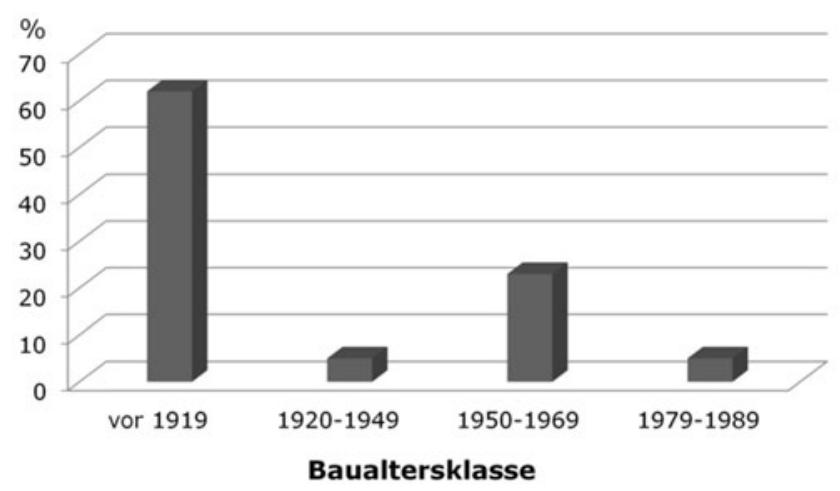

Abb. 4 Von Fortzügen in ein neues Baugebiet betroffene Baugebiete nach Baualtersklassen. (Quelle: Eigene Erhebung)

Als weiteres Ergebnis konnte eine zweite innerörtliche ,Umzugswelle' festgestellt werden, da die durch die ersten Umzüge frei gewordenen Gebäude wenigstens zum Teil von Einwohnern der peripheren Ortsteile nachgenutzt wurden (vgl. Abb. 5, gebäudescharfe Darstellung aus Datenschutzgründen nicht möglich). Diese Konzentration in der Kerngemeinde wurde begünstigt durch finanzielle Förderung wie dem Stadtumbauprogramm des Landes Hessen.

\subsection{Innenentwicklungspotenzial als Voraussetzung für Zentrierung}

Die für die Schaffung einer kompakten Siedlung zu unterstützende (Re)Zentrierung der Einwohner ist nur möglich, wenn in der zentralen Siedlung genügend Innenentwicklungspotenzial vorhanden ist. Die Feststellung dieses Potenzials ist jedoch mit Unsicherheiten verbunden. „Innenentwicklungspotentiale fallen selten in größerer Anzahl zu einem bestimmten Zeitpunkt an" (Dahm 2007: 60), sondern tauchen vielmehr in unregelmäßigen Abständen, beispielsweise nach Schließungen größerer Unternehmen oder aber auch durch die Altersentwicklung der Bevölkerung und dadurch bedingten Erbschafts- und Verkaufsfällen mit einer gewissen Kontinuität auf. In Haufendörfern ist das ,theoretische Innenentwicklungspotential“ (Bleher/Buchert 2008: 56) anhand einer Abfrage

- der Baulücken: Das Grundstück liegt innerhalb der im Zusammenhang bebauten Ortsteile oder im Geltungsbereich eines Bebauungsplanes, direkt angrenzend an eine öffentliche Erschließungsstraße, die Größe beträgt mindestens $350 \mathrm{~m}^{2},{ }^{6}$

- der geringfügig genutzten Flächen: Lage innerhalb der im Zusammenhang bebauten Ortsteile oder im Geltungsbereich eines Bebauungsplanes und mit einer Grundflächenzahl $<0,1$,

\footnotetext{
${ }^{6}$ Kleinste Grundstücksfläche für eine Doppelhaushälfte laut Amt für Bodenmanagement (2007: 56); ähnliche Festlegungen bezüglich der Flächengröße bei Preuß und Floeting (2009: 63).
} 
Abb. 5 Unterteilung einer Gemeinde in Baualtersklassen und innerörtliche Wanderungen aus diesen Gebieten in ein Neubaugebiet nach dessen Ausweisung. (Quelle: Eigene Erhebung auf Basis des Web Map Services (WMS) des HLBG, vgl. http:// www.gds-srv.hessen.de/cgi-bin/ lika-services/ogc-free-maps.ows? (27.08.2014))

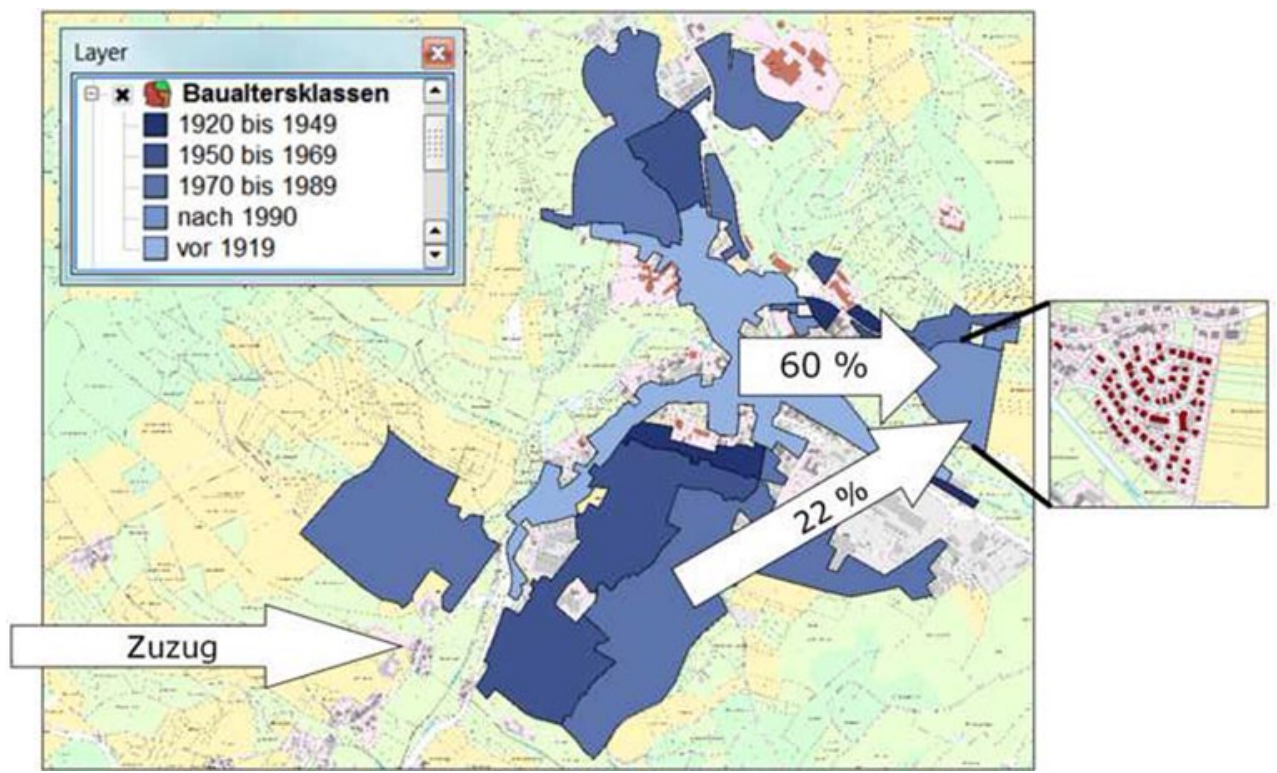

- der Brachflächen und leer stehenden Gebäude: Für Wohngebäude über das Melderegister, für gewerbliche Flächen mithilfe des Gewerberegisters und durch Überschneidung der Daten aus dem ALKIS nach der Objektkennung für die Nutzung

feststellbar. Stets ergänzend ist das Wissen der Einwohner und insbesondere der Kommunalpolitiker einzubeziehen, da beispielsweise landwirtschaftlich genutzte Gebäude weder im Melde- noch im Gewerberegister geführt werden. Das theoretische Potenzial entspricht zudem aus diversen Gründen wie stadtökologischer Aspekte, unterschiedlicher Eigentümerinteressen oder der spezifischen kommunalpolitischen Interessenlagen und Kapazitäten nicht dem tatsächlichen Bauland.

In einer Gemeinde im Untersuchungsgebiet stehen heute beispielsweise durchschnittlich $8 \%$ der Wohngebäude leer (mit großen Schwankungen zwischen den Ortsteilen sowie den Baualtersklassen, vgl. Abb. 6), etwa ein Fünftel der bebauten Grundstücke sind mindergenutzt. Die Gesamtfläche der unbebauten, aber grundsätzlich für eine Wohnbebauung nutzbaren Grundstücke, wurde auf etwa 22 ha bilanziert.

In Dörfern mit der Siedlungsstruktur von Anger-, Straßendörfern oder Einzelhofreihungen gestaltet sich die Feststellung des Innenentwicklungspotenzials deutlich schwieriger als in Haufendörfern. Eine Nachverdichtung ist in vielen Teilen solcher Gemeinden einer Neuausweisung gleichzusetzen, die oben genannten Abfragekriterien greifen nicht. Hier ist fast ausschließlich auf die tatsächliche Vorortsituation abzustellen.

In einem zweiten Schritt sollte eine Wohnbedarfsprognose aufgrund der vorliegenden Bevölkerungsvorausberechnungen, der bisherigen Entwicklungen und der zu

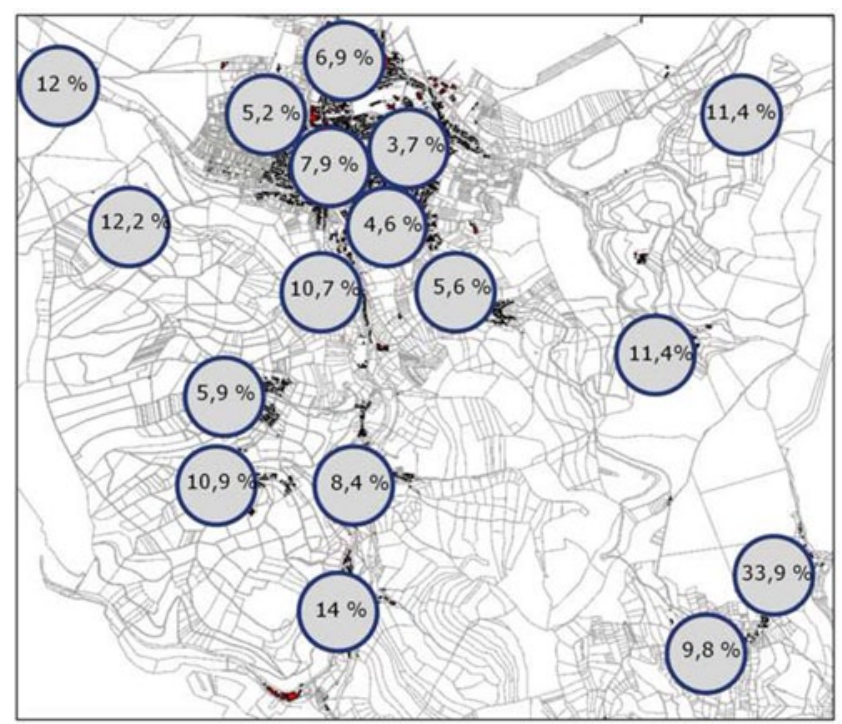

Abb. 6 Heutige Leerstandsraten (prozentualer Anteil nicht genutzter Wohneinheiten am Gesamtbestand der Wohneinheiten) einer Untersuchungsgemeinde. (Quelle: Eigene Erhebung, Darstellung auf Basis der ALKIS-Daten des HLBG sowie des Melderegisters der Gemeinde)

erwartenden Nachfrage bezüglich Standards abgeleitet werden. Geht man davon aus, dass die Bevölkerung im Untersuchungsgebiet in den kommenden zehn Jahren dem Trend entsprechend um weitere $2 \%$ zurückgeht (Hessen Agentur 2010: 38) und der Bedarf an Wohnfläche pro Kopf (im Untersuchungsgebiet $47 \mathrm{~m}^{2}$ ) stagniert, ist hier mit einem Rückgang um etwa 12 ha zu rechnen. Das mithilfe des GIS aufgezeigte Innenentwicklungspotenzial von 22 ha könnte demnach - zumindest theoretisch - den Bedarf an anderem als dem bestehenden Wohnraum decken. 


\subsection{Verbindung von Abfallplanung und Siedlungsentwicklung}

Für eine strategische Planung ließen sich die bisher vorgestellten Untersuchungen vereinen und gegenseitig nutzen. Die in Kap. 3.2 dargestellte Umstellung von Hol- auf Bringsystem in der Abfallentsorgung könnte abhängig gemacht werden von der tatsächlichen Schüttmenge und Behälterdichte sowie von deren zu erwartender zukünftiger Entwicklung. Das Holsystem könnte auf dichter besiedelte Ortsteile beschränkt und in weniger dicht besiedelten Teilen auf das Bringsystem umgestellt werden. Straßenzüge mit wenig Leerstand würden auch zukünftig von den Sammelfahrzeugen wie bisher bedient, in den Straßen, die einen hohen Leerstandsanteil haben, würden die Anwohner verpflichtet, ihren Abfall zu Sammelstellen zu bringen. Aufgrund der geringen Menge Restmüll pro Kopf ist zu erwarten, dass dies auch älteren Menschen möglich ist (entsprechend zu Altglas und Papier), wobei die Machbarkeit im Einzelfall geprüft werden sollte. Allerdings ist im Hinblick auf Studien zur Akzeptanz von verursachergerechten Abfallgebühren darauf hinzuweisen, dass eine Änderung des Tagesablaufes eine geringere Akzeptanz erwarten lässt, der finanzielle Anreiz nicht mehr steigender Abfallgebühren dies jedoch - zumindest teilweise - ausgleichen kann (Schütz 1991; IÖR 2001). Entscheidend für die Akzeptanz ist die Sensibilisierung für die Notwendigkeit der Umstellung (IÖR 2001: 33).

Durch die teilweise Aufgabe des Sammelns im Holsystem würde die Abfallentsorgung nicht nur kostengünstiger werden, sondern könnte gleichzeitig als Steuerungsmittel der Siedlungsentwicklung dienen. Der Benutzerkomfort wäre für die Betroffenen selbstredend eingeschränkt, gerade diese Einschränkung wäre als weicher Standortfaktor auch gleichzeitig Einflussfaktor für die Zuwanderung bzw. den Neubezug von Immobilien. Die nicht mehr angeschlossenen Straßenzüge verlieren an Attraktivität. Sollen nun einzelne Quartiere zur Entwicklung einer kompakten Siedlung nicht weiterentwickelt werden, kann dieser Attraktivitätsverlust von der Gemeinde gezielt eingesetzt werden. So würde der Abfallservice als Instrument der räumlichen Planung die oben beschriebene Zentrierung unterstützen. Politisch gesehen ist die Wirksamkeit sicherlich zu relativieren, da ein solches Vorgehen als zu großer Eingriff in das private Eigentum gewertet werden wird, wird doch die Wertminderung von Immobilien regelrecht aufgezwungen.

Anhand der Daten eines Stadtteils mit 373 Einwohnern (Stand 2010) mit einer Entfernung vom benachbarten Stadtteil von etwa sechs Kilometern und einer Stichstraße als einzigem Zugang wurde eine Beispielrechnung durchgeführt. Der Stadtteil gehört zur Stadt Erbach im Odenwald. Zurzeit steht eine ungefähre Transportzeit (Weg in den Ortsteil) von acht Minuten einer Sammelzeit von 61 min (errechnet

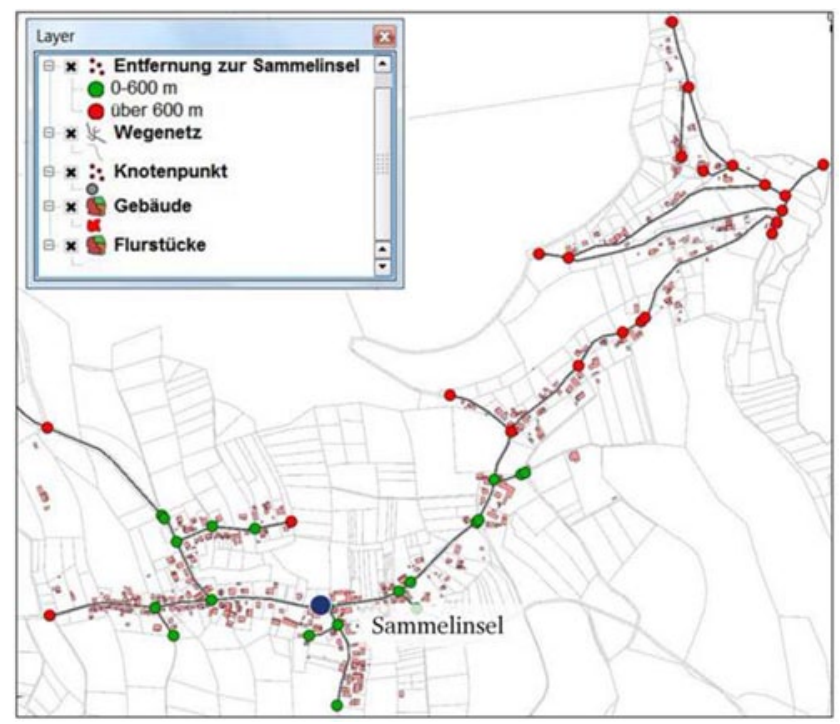

Abb. 7 Erreichbarkeit einer Sammelinsel. (Quelle: Eigene Darstellung auf Basis der ALKIS-Daten des HLBG)

aus der heutigen Tonnendichte von $2,5 \mathrm{t}$ je $100 \mathrm{~m}$ Straße) gegenüber. Über $10 \%$ der Einwohner über 70 Jahre leben alleine, der überwiegende Teil der Gebäude gehört zu den Baualtersklassen bis 1919, 1920er bis 1940er Jahre und 1950er bis 1960er Jahre. Der Ortsteil weist bereits heute eine Leerstandsquote von etwa $18,6 \%$ auf, wobei im nördlichen Teil eine Leerstandsquote von etwa $33 \%$ erreicht wird. In der Zusammenschau der funktionalen und strukturellen sowie der demographischen Merkmale (vgl. dazu ausführlich Köhler 2011) ist mit vernachlässigbaren Wanderungsgewinnen zu rechnen. In Verbindung mit der Sterbetafel und den Einwohnermeldedaten ergibt sich für das Jahr 2025 eine Verringerung der Tonnendichte auf durchschnittlich 1,9 t pro $100 \mathrm{~m}$. Die Sammelzeit wird sich rein rechnerisch jedoch um lediglich etwa elf Minuten verringern. ${ }^{7} \mathrm{Da}-$ bis auf eine Ausnahme - alle Straßen Stichstraßen sind und die Straßenbreite durchschnittlich $9,5 \mathrm{~m}$ beträgt, sind die logistischen Voraussetzungen zudem nicht optimal. Zukünftig könnte die Restmüllentsorgung in diesem Stadtteil deshalb im Bringsystem erfolgen.

Auch hier wurde deshalb der Standort für eine Sammelinsel vorgeschlagen (vgl. Abb. 7). Allerdings liegt diese aufgrund örtlicher Gegebenheiten nicht in fußläufiger Erreichbarkeit aller Einwohner. Eine zweite Sammelinsel rentiert sich jedoch wegen des geringen Abfallaufkommens nicht. Die betroffenen Anwohner müssten ihren Restmüll über eine weitere Strecke transportieren, beispielsweise zum nächsten Wertstoffhof (etwa $12 \mathrm{~km}$ ). Bei einem solchen Eingriff in das bisherige Entsorgungssystem ist auf die

\footnotetext{
${ }^{7}$ Bei einem dem Trend entsprechenden durchschnittlichen Bevölkerungsrückgang in der Gemeinde um $2 \%$ und konstanter Schüttmenge pro Kopf.
} 
Bedeutung eines funktionierenden Gemeinschaftssystems oder die Etablierung privater Dienste (wie z. B. DORV-Zen$\operatorname{tren}^{8}$ ) hinzuweisen: Ebenso wie die Versorgung mit Lebensmitteln, Medikamenten und ärztlicher Betreuung könnte bei immobilen Menschen die Möglichkeit zur Entsorgung des Restmülls im Bringsystem so gewährleistet werden.

Mit Einführung des Bringsystems würde das betroffene Quartier unattraktiver und könnte bei weiterer ,Entleerung ${ }^{\text {‘ }}$ im Sinne eines Siedlungsumbaus schlussendlich rückgebaut werden. Diese Überlegungen betreffen sehr langfristige Entwicklungen und implizieren keine Zwangsumsiedlungen. Zudem fehlen bisher planungsrechtliche Umsetzungsmöglichkeiten, über die beispielsweise ein Zuzugsverbot ausgesprochen werden könnte.

Folgende Kriterien zur Entscheidung über Bring- oder Holsystem werden vorgeschlagen:

- Abfallaufkommen (Tonnendichte über Einwohnerdichte), zu ermitteln über Einwohnermelde- und Routingdaten,

- Höhe der Leerstandsquote (ergibt sich aus Meldedaten, ALKIS, aber auch aus Ortsbegehungen und Befragungen); nur in Gebieten mit sehr hohem Leerstandsanteil ist die Abkopplung vom bisher angebotenen Holsystem sozialstaatlich denkbar,

- Lange Anfahrtswege für die Sammelfahrzeuge im Verhältnis zu den Bedienten (Transportzeit zu Sammelzeit bzw. Anteil des Abfallaufkommens),

- Logistische Voraussetzungen: Stichstraßen ohne Wendemöglichkeit und Straßen mit einer Breite unter neun Metern.

Die Entscheidung über die Anbindung an das Holsystem oder die Abkopplung kann allerdings nur von den Kommunen selbst - einschließlich der Einwohner - erarbeitet und getroffen werden.

\section{Fazit und Ausblick}

Für eine nachhaltige räumliche Planung, die die Schaffung einer kompakten Siedlung trotz Schrumpfungsbedingungen zum Ziel hat, sollten raumbezogene Rahmendaten wie Leerstandsrate, Leerstandsrisiko, Innenentwicklungspotenzial, bevorzugter Wohnort, Wohnbedarf etc. bekannt sein. Geodaten in Verknüpfung mit beschreibenden Daten liefern hier gute Grundlagen und ermöglichen eine Visualisierung von Entwicklungen. Frei verfügbare Daten wie OpenStreetMap (OSM)-Daten und Schüttmengen allein sind dafür unter Umständen allerdings nicht ausreichend und müssen um teils sensible Daten ergänzt werden. Für die planende

${ }^{8}$ DORV: Dienstleistung und Ortsnahe Rundum Versorgung; http:// www.dorv.de (27.08.2014).
Gemeinde ist dies jedoch grundsätzlich rechtlich unproblematisch, da sie die datenhaltende Behörde ist. Lediglich für Dritte (z. B. Planungsbüros) muss eine Regelung zu Zugangsmöglichkeiten, z. B. durch Anonymisierung, gefunden werden.

Durch die Verknüpfung der Meldedaten und der Adresssätze der Abfallentsorgung konnten die beiden Datensätze verglichen und so Fehler identifiziert werden, da jeder Haushalt über eine Restmülltonne verfügen muss. Im Zuge der Untersuchung zeigten sich Fehler in den beiden Datenbeständen, was als Nebeneffekt zu einer Identifizierung von gemeldeten Personen ohne Restmülltonne führte. Gleichzeitig wurde deutlich, dass auch bei gemeindlichen Daten weiterer Harmonisierungsbedarf besteht und Ortsbegehungen oft unvermeidbar waren. Durch die wachsende Menge an Daten, beispielsweise über die Geodateninfrastruktur Deutschland (GDI-DE) ${ }^{9}$, werden sich voraussichtlich zukünftig jedoch auch deren Qualität und Verfügbarkeit verbessern.

Im Ergebnis kann sich die Verknüpfung von räumlicher Gesamtplanung und Abfallentsorgungsservice als sinnvoll erweisen, wobei durch den Einsatz von Geodaten aufgezeigt werden kann, wo Entwicklung möglich ist, wo eine Umstellung auf das Bringsystem technisch sinnvoll sein kann und wo eine Sammelinsel platziert werden kann. Die zum Teil herausfordernde Datensituation würde jedoch eine Einbeziehung von Ortsbegehungen, Befragungen etc. für eine tatsächliche Umsetzung der Maßnahmen unabdingbar machen. Unabhängig von den technischen und quantitativ messbaren Voraussetzungen sind dabei der gesellschaftliche Konsens, ein großer politischer Wille sowie eine starke Bürgergemeinschaft zur Umsetzung und eine einzelfallbezogene Untersuchung der sozialen Verträglichkeit notwendig. Zudem ist die zeitliche Komponente zu beachten, da sowohl eine überstürzte Einführung des Bringsystems als auch die kurzfristige Konzeption der Rückbaugebiete systematische Fehler darstellen könnten, die von vornherein eine Umsetzung verhindern.

\section{Literatur}

Amt für Bodenmanagement (2007): Grundstücksmarktbericht Südhessen. Daten des Immobilienmarktes Südhessen. Wiesbaden.

Beetz, S. (2012): Besonderheiten in der Entwicklung kleiner Städte in ländlichen Räumen. In: Engel, A.; Harteisen, U.; Kaschlik, A. (Hrsg.): Kleine Städte in peripheren Regionen - Prozesse, Teilhabe und Handlungsbefähigung, integriertes Stadtentwicklungsmanagement. Detmold, 45-66.

Berndgen-Kaiser, A.; Bläser, K.; Fox-Kämper, R.; Siedentop, S.; Zakrzewski, P. (2014): Demography-driven suburban decline? At the crossroads: mature single-family housing estates in Germany. In: Journal of Urbanism 7 (3), 286-306.

${ }^{9} \mathrm{http}: / / \mathrm{www}$. geoportal.de/DE/GDI-DE/gdi-de.html?lang = de (30.09.2014). 
Bilitewski, B. (2007): Verursachergerechte Gebührensysteme im Vergleich. Aufwand, Kosten, Nutzen. Vortrag vom 20. November 2007, Praxisforum Verursachergerechte Gebührensysteme in der Praxis. Hamburg.

Bilitewski, B.; Härdtle, G.; Marek, K. (2000): Abfallwirtschaft Handbuch für Praxis und Lehre. Berlin, Heidelberg, New York.

Bleher, D.; Buchert, M. (2008): Die Zukunft liegt im Bestand. Innenentwicklung als unterschätzte Chance für kleine und große Gemeinden. Ergebnisse des Forschungsprojektes komreg. In: Institut für Wasserbau der Universität Stuttgart (Hrsg.): VEGAS-Kolloquium 2008. Ressource-Fläche III. Tagungsband zur Veranstaltung am 01. Oktober 2008 an der Universität Stuttgart. Stuttgart, 55-63. = Mitteilungen des Instituts für Wasserbau 174.

Cord-Landwehr, K. (2002): Einführung in die Abfallwirtschaft. Stuttgart.

Dahm, S. (2007): Bau- und Wohnflächenreserven in kleinen Kommunen Baden-Württembergs - Innenentwicklungspotenziale vor dem Hintergrund einer sich ändernden Bevölkerungsstruktur. Karlsruhe $=$ Schriftenreihe des Instituts für Städtebau und Landesplanung der Universität Karlsruhe 36.

Förstner, U. (2008): Umweltschutztechnik. Berlin, Heidelberg.

Hagemeister, U.; Haller, C. (2009): Bürgermitwirkung im Stadtumbau. In: Altrock, U.; Kunze, R.; Pahl-Weber, E.; von Petz, U.; Schubert, D. (Hrsg.): Jahrbuch Stadterneuerung 2009. Schwerpunkt „Megacities und Stadterneuerung“. Berlin, 261-280.

Hahne, U. (2013): Auswirkungen des Demographischen Wandels auf den Immobilienmarkt in ländlichen Räumen. In: Rüppel, U. (Hrsg.): 2. Darmstädter Ingenieurkongress Bau und Umwelt. Aachen, 339-344.

Hessen Agentur (2010): Bevölkerungsvorausschätzung für die hessischen Landkreise und kreisfreien Städte. Eine Projektion für den Zeitraum von 2010 bis 2030 und eine Trendfortschreibung bis 2050. Wiesbaden.

Hessischer Rechnungshof (2009): Neunzehnter Zusammenfassender Bericht 2009. Darmstadt.

Hessisches Statistisches Landesamt (2013): Hessische Kreiszahlen. Ausgewählte neue Daten für Landkreise und kreisfreie Städte. Band 1, 2013. Wiesbaden.

ILS - Institut für Landes- und Stadtentwicklungsforschung; Planersocietät (2009): Stadtumbau und Infrastruktursysteme in NordrheinWestfalen - Wege zur kosteneffizienten Anpassung des Bestandes. Dortmund.

IÖR - Leibniz-Institut für ökologische Raumentwicklung (2001): Chancen und Barrieren eines verursachergerechten Abfallgebührensystems in der Individualisierungsgesellschaft. Dresden.

Köhler, T. (2011): Verwaltungsreform im ländlichen Raum. Ein Diskussionsbeitrag. Darmstadt. = Schriftenreihe des Geodätischen Instituts der Technischen Universität Darmstadt 34.

Kötter, T. (2009): Innenentwicklung der Dörfer und Revitalisierung der Dorfkerne - von der Dorferneuerung zum Dorfumbau. In: DLKG - Deutsche Landeskulturgesellschaft (Hrsg.): Dörfer ohne Menschen!? Zwischen Abriss, Umnutzung und Vitalisierung. o.O., 6-28. = Schriftenreihe der Deutschen Landeskulturgesellschaft, Sonderheft 02.

Linke, H. J.; Köhler, T. (2010): Ansätze zum Siedlungsumbau bei sinkenden Bevölkerungszahlen im ländlichen Raum. In: Flächenmanagement und Bodenordnung 72 (3), 102-109.
Miofsky, D.; Souren, R. (2010): Einflussfaktoren auf die Höhe der Hausmüllgebühren. Ilmenau. = Ilmenauer Schriften zur Betriebswirtschaftslehre $2 / 2010$.

OREG - Odenwald-Regional-Gesellschaft (2012): Nahverkehrsplan des Odenwaldkreises. Schlussbericht in der Fassung des Kreistagsbeschlusses vom 25. Juni 2012. Erbach. http://www. odenwaldmobil.de/uploads/media/Nahverkehrsplan_2013-17.pdf (27.08.2014).

Preuß, T.; Floeting, H. (2009): Folgekosten der Siedlungsentwicklung. Bewertungsansätze, Modelle und Werkzeuge der Kosten-NutzenBetrachtung. Berlin. = Beiträge aus der REFINA-Forschung 3 .

Prinz, D. (1999): Städtebau. Band 1: Städtebauliches Entwerfen. Stuttgart.

Rahmeyer, F. (2004): Abfallwirtschaft zwischen Entsorgungsnotstand und Überkapazitäten. Augsburg. = Volkswirtschaftliche Diskussionsreihe Universität Augsburg, Beitrag 266.

Renner, M. (2007): Mitwirkung der Bürgerinnen und Bürger an der Stadtentwicklung - ein Überblick mit Beispielen aus Projekten. In: Informationen zur Raumentwicklung 1, 1-16.

Salhofer, S. (2001): Kommunale Entsorgungslogistik. Planung, Gestaltung und Bewertung entsorgungslogistischer Systeme für kommunale Abfälle. Berlin. = Abfallwirtschaft in Forschung und Praxis 121.

Schaffert, M. (2011): Szenariotechnik und GIS - Ein Beitrag zur demographierobusten Planung in Kommunen. Darmstadt. = Schriftenreihe des Geodätischen Instituts der Technischen Universität Darmstadt 32.

Scherf, C. (2007): Der sozioökonomische Wandel in Schrumpfungsräumen und Folgen für den Wirtschaftsverkehr. Berlin. = IVPSchriften 14.

Schiller, G.; Siedentop, S. (2005): Infrastrukturfolgekosten der Siedlungsentwicklung unter Schrumpfungsbedingungen. In: DISP $160,83-93$.

Schmied, D. (2007): Leerstände in Dörfern. Ausmaß und Ursachen. In: Schmied, D.; Henkel, G. (Hrsg.): Leerstand von Gebäuden in Dörfern - Beginn der Dorfauflösung oder Chancen durch Umnutzung? Göttingen, 1-18.

Schmied, D.; Henkel, G. (Hrsg.) (2007): Leerstand von Gebäuden in Dörfern - Beginn der Dorfauflösung oder Chancen durch Umnutzung? Göttingen.

Schöfl, G.; Schöfl, M.; Speidel, S. (2009): Kommunales Flächenmanagement im Ländlichen Raum - die Aktivierung ungenutzter Gebäude und Bauflächen am Beispiel MELAP. In: Flächenmanagement und Bodenordnung 71 (6), 271-276.

Schütz, H. (1991): Individuelles Entsorgungsverhalten und Akzeptanz von Entsorgungstechnologien. Jülich. = Arbeiten zur Risiko-Kommunikation 22.

Siebel, W. (2006): Wandel, Rationalität und Dilemmata der Planung. In: PND online 4, 1-22.

Statistisches Bundesamt (2014): Umwelt - Zeitreihe zum Abfallaufkommen. https://www.destatis.de/DE/ZahlenFakten/GesamtwirtschaftUmwelt/Umwelt/UmweltstatistischeErhebungen/Abfallwirtschaft/ Tabellen/ZeitreiheAbfallaufkommen.pdf?_blob=publicationFile (27.08.2014). 